\title{
Levantamento da ictiofauna da Floresta Nacional de Canela, na região superior da bacia hidrográfica do Rio Caí, Rio Grande do Sul, Brasil
}

\author{
Renato Bolson Dala-Corte ${ }^{1,3}$, Ismael Franz ${ }^{1}$, Marcelo Pereira de Barros ${ }^{1}$ \& Paulo Henrique Ott ${ }^{2}$ \\ ${ }^{1}$ Laboratório de Zoologia, Instituto de Ciências da Saúde, Centro Universitário Feevale, \\ Campus II, RS-239, 2755, CEP 93352-000, Novo Hamburgo, RS, Brasil, e-mail: barrospm@cpovo.net \\ ${ }^{2}$ Universidade Estadual do Rio Grande do Sul - UERGS, Pólo Cidreira, \\ Av.Mostardeiro, 3635, CEP 95595-000, Cidreira, RS, Brasil,e-mail: paulo-ott@uergs.edu.br \\ ${ }^{3}$ Autor para correspondência: Renato Bolson Dala Corte, e-mail: renatocorte@gmail.com
}

DALA-CORTE, R.B., FRANZ, I., BARROS, M.P. \& OTT, P.H. 2009. A survey of the ichthyofauna at Floresta Nacional de Canela, in the upper region of Rio Caí basin, Rio Grande do Sul, Brazil. Biota Neotrop., 9(2): http://www.biotaneotropica.org.br/v9n2/en/abstract?inventory+bn01709022009.

\begin{abstract}
Data referring to a survey of the fish fauna at Floresta Nacional de Canela, a National Conservation Unity located in the upper region of Rio Caí basin, in higher altitudes of Serra Geral do Rio Grande do Sul are presented in this study. Nine sites were sampled in several aquatic environments, including a swamp, dams and streams situated inside and in the nearby area of the unity. In order to capture the specimens, hand net, seine net, fishing lines, gill nets and baited traps were used. Altogether 20 species were found, belonging to 10 families and 6 orders. Hyphessobrycon luetkenii had a wider distribution, occurring in almost all of the sampled points, while Callichthys callichthys was recorded in only one site. In the dams, the order Perciformes was the most representative, followed by Cypriniformes and Characiformes. These environments are characterized by the dominance of family Cichlidae (Perciformes), of which most of the Neotropical species have preference for stagnant water environments. The occurrence of four exotic introduced species is highlighted (Ctenopharingodon idella, Cyprinus carpio, Hypophthalmichthys molitrix and Micropterus salmoides) with distribution restricted to the dams. For the streams, a greater specific richness of Characiformes and Siluriformes of small size was observed, a pattern that had already been found in the South American streams. It is also featured, the presence of species with non defined taxonomic status, such as Trichomycterus sp. and Astyanax aff. scabripinnis, reinforcing the importance of surveys on headwaters streams from Rio Grande do Sul, in order to obtain information to subsidize measures to conserve these environments. Despite the fact that the exotic species were registered only in the dams, their eradication from the conservation unity is recommended, considering the threats that they may represent for the native ichthyofauna and for the other aquatic ecosystems of the region.
\end{abstract}

Keywords: fish, headwaters streams, National Conservation Unity, exotic species.

DALA-CORTE, R.B., FRANZ, I., BARROS, M.P. \& OTT, P.H. 2009. Levantamento da ictiofauna da Floresta Nacional de Canela, na região superior da bacia hidrográfica do Rio Caí, Rio Grande do Sul, Brasil. Biota Neotrop., 9(2): http://www.biotaneotropica.org.br/v9n2/pt/abstract?inventory+bn01709022009.

Resumo: Neste trabalho são apresentados dados referentes a um inventário da fauna de peixes da Floresta Nacional de Canela, uma Unidade de Conservação Federal localizada no trecho superior da bacia hidrográfica do Rio Caí, em elevadas altitudes da Serra Geral do Rio Grande do Sul. Foram amostrados nove pontos de coleta em diversos ambientes aquáticos, incluindo um banhado, açudes e arroios, situados tanto no interior quanto no entorno da unidade. Para a captura dos exemplares utilizou-se puçá, picaré, linhas de pesca, redes de emalhar e armadilhas do tipo covo. Ao total, foram encontradas 20 espécies pertencentes a 10 famílias e 6 ordens. Hyphessobrycon luetkenii teve distribuição mais ampla, ocorrendo em quase todos os pontos amostrados, enquanto Callichthys callichthys foi registrado somente em um ponto. Nos açudes, a ordem Perciformes foi a mais representativa, seguida por Cypriniformes e Characiformes. Esses ambientes caracterizaram-se pela predominância da família Cichlidae (Perciformes), cuja maioria das espécies Neotropicais tem preferência por ambientes de água parada. Com distribuição restrita aos açudes, destaca-se a ocorrência de 4 espécies exóticas introduzidas (Ctenopharingodon idella, Cyprinus carpio, Hypophthalmichthys molitrix e Micropterus salmoides). Para os arroios, foi observado maior riqueza específica de Characiformes e Siluriformes de pequeno porte, padrão já encontrado em muitos estudos nos riachos sul-americanos. Destaca-se a presença de espécies com "status" taxonômico indefinido, tais como Trichomycterus sp. e Astyanax aff. scabripinnis, reforçando a importância dos levantamentos em riachos nas cabeceiras de rios do Rio Grande do Sul, para a obtenção de informações que subsidiem medidas de conservação desses ambientes. Embora as espécies exóticas tenham sido registradas apenas em açudes, recomenda-se a erradicação destas da unidade de conservação, em função das ameaças que podem representar à ictiofauna nativa e aos demais ecossistemas aquáticos da região.

Palavras-chave: peixes, riachos de cabeceira, Unidade de Conservação Federal, espécies exóticas. 


\section{Introdução}

A região neotropical contém a maior diversidade de peixes de água doce de todo o planeta (Vari \& Malabarba 1998). No entanto, o conhecimento dessa ictiofauna ainda é limitado e incompleto (Bohlke et al. 1978, Lowe-McConnell 1999, Reis et al. 2003a) e, segundo Schaefer (1998), o número de espécies de peixes de água doce para o Neotrópico pode chegar a 8.000 , o que representaria cerca de $25 \%$ de todas as espécies de peixes do mundo (Vari \& Malabarba 1998).

No Brasil, várias atividades antrópicas vêm alterando as condições naturais dos ambientes aquáticos, representando sérias ameaças à diversidade de peixes. De acordo com Agostinho et al. (2005), as principais ameaças aos ecossistemas aquáticos continentais brasileiros são a poluição, o desmatamento, a construção de barragens, a pesca predatória e a introdução de espécies exóticas. Segundo ainda os mesmos autores, esses problemas são mais conspícuos nas regiões mais desenvolvidas do Brasil, ou seja, no sudeste e sul do país.

A hidrografia do Rio Grande do Sul pode ser dividida em 3 sistemas principais: Rio Uruguai, Rio Tramandaí e Laguna dos Patos. Para o Estado, como um todo, estão descritas 262 espécies de peixes de água doce, além de aproximadamente mais de 60 já conhecidas, mas ainda não descritas formalmente (Reis et al. 2003b). Deste total, 121 espécies são conhecidas para o sistema da laguna dos Patos. Acredita-se que esses números provavelmente mudem à medida que os estudos taxonômicos avancem e que novos inventários sejam realizados (Becker et al. 2006).

Situada no sistema da Laguna dos Patos, sendo um dos principais tributários do lago Guaíba, a bacia hidrográfica do Rio Caí apresenta uma das áreas mais densamente povoadas do RS. Desse modo, a área da bacia encontra-se sujeita a uma série de impactos ambientais, podendo-se destacar as barragens localizadas no curso superior do rio e a diluição de efluentes provenientes de esgotos domésticos e industriais (SEMA-RS, 2008).

O conhecimento da distribuição das espécies de peixes do Rio Grande do Sul e o inventariamento de regiões pouco avaliadas, como riachos nas cabeceiras dos principais rios, estão entre os estudos prioritários para que se tenham informações que subsidiem medidas de preservação da ictiofauna do Estado (Reis et al. 2003b). As drenagens da Bacia do Caí são pobremente estudadas quanto a sua ictiofauna, principalmente nas regiões de cabeceiras. A maior parte das informações disponíveis na literatura referem-se ao trecho inferior do rio (Hensel 1868, 1870, Teixeira 1989, Volkmer-Ribeiro et al. 2004).

Nesse contexto, as Unidades de Conservação podem desempenhar um papel importante em estratégias de preservação. A Floresta Nacional (FLONA) de Canela é a maior Unidade de Conservação localizada na região do curso superior da bacia hidrográfica do Rio Caí e, implantada há mais de 50 anos, ainda não possui um levantamento de sua ictiofauna.

O presente estudo apresenta dados referentes à inventariamentos da fauna de peixes da FLONA de Canela e da região de entorno, com a intenção de contribuir para a atualização do seu plano de manejo e para um maior conhecimento da fauna de peixes da região.

\section{Material e Métodos}

\section{1. Área de estudo}

A FLONA de Canela foi criada em 1946 pelo extinto Instituto Nacional do Pinho e está localizada a $6 \mathrm{~km}$ do centro do município de Canela, RS (Ferraz 2003). A unidade de conservação enquadra-se na categoria de "Uso Sustentável", a qual visa conciliar a utilização dos recursos naturais com a preservação ambiental (SNUC 2002).
A unidade localiza-se na região do planalto das araucárias do Rio

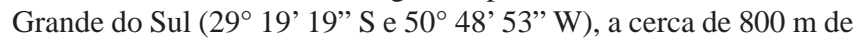
altitude, onde a formação vegetal é denominada Floresta Ombrófila Mista (Teixeira et al. 1986). Sua área compreende um total de 517 ha, dos quais 129 ha representam áreas de floresta nativa, 281 ha de silvicultura (com plantações de Araucaria angustifolia, Pinus spp. e Eucalyptus spp.), 20 ha de banhados e drenagens e 87 ha de áreas abertas ou construídas (Schneider et al. 1989).

De acordo com a classificação climática de Köppen, o clima do município de Canela é do tipo $\mathrm{Cfb}$, temperado úmido, com chuvas ocorrendo durante todos os meses do ano, temperatura média do mês mais quente inferior a $22{ }^{\circ} \mathrm{C}$ e temperatura média anual inferior a $18^{\circ} \mathrm{C}$ (Moreno 1961). No inverno, geadas e nevoeiros são freqüentes (Fortes 1959).

Em relação às drenagens e demais ambientes aquáticos, a FLONA de Canela está inserida na região do curso superior da bacia hidrográfica do Rio Caí, sendo drenada pelos arroios Caçador e Tiririca (Figura 1), 2 tributários de pequeno porte do Rio Caí. No interior da unidade é comum a ocorrência de pequenos cursos d'água que drenam para estes arroios, além de açudes e banhados. Na região do entorno da unidade, destaca-se o arroio Saiqui, outro tributário do Rio Caí, localizado a montante em relação aos arroios Caçador e Tiririca.

\section{Pontos de amostragem}

As amostragens abrangeram diversos ambientes aquáticos, situados tanto no interior quanto no entorno da unidade, incluindo nove pontos de coleta (Figura 1), descritos na caracterização a seguir:

- Arroio Caçador (pontos P1 e P2) - Possui em torno de $12 \mathrm{~km}$ de extensão e deságua no Rio Caí formando a cascata Caçador, cuja altura aproximada é de $80 \mathrm{~m}$. Os 2 pontos amostrados apresentam alto grau de sombreamento devido à mata ciliar e estão situados a montante da cascata Caçador. No ponto $\mathrm{P} 1\left(29^{\circ} 18^{\prime} 50^{\prime \prime} \mathrm{S}\right.$ e $\left.50^{\circ} 47^{\prime} 17^{\prime \prime} \mathrm{W}\right)$ predominam corredeiras com água rasa e leito constituído por rochas. No ponto P2 $\left(29^{\circ} 18^{\prime} 20^{\prime}\right.$ ' S e 50 48' $\left.06^{\prime \prime} \mathrm{W}\right)$ ocorre um afloramento rochoso com pouca correnteza, profundidade média de $1 \mathrm{~m}$ e leito com matéria orgânica acumulada.

- Arroio Tiririca (pontos P3 e P4) - Possui aproximadamente $8 \mathrm{~km}$ de extensão, percorre trechos em áreas urbanas e é um afluente do arroio Caracol, o qual deságua no Rio Caí formando a cascata Caracol (aproximadamente $131 \mathrm{~m}$ de altura). O ponto P3 (29 $19^{\prime} 33^{\prime}$ ' S e 50 48' 31' W) apresenta alto grau de sombreamento devido à mata ciliar (presença de Pinus spp.), predomínio de corredeiras com água rasa e leito constituído por rochas. O ponto $\mathrm{P} 4$ (29 $19^{\circ}$ ' $01^{\prime \prime} \mathrm{S}$ e $\left.50^{\circ} 51^{\prime} 07^{\prime \prime} \mathrm{W}\right)$ localizase próximo a foz, onde a mata ciliar é escassa, a correnteza é moderada, a profundidade média é de $0,8 \mathrm{~m}$ e o leito é constituído por pedras de diferentes tamanhos e areia.

- Arroio Saiqui (ponto P5) - Possui em torno de 7 km de extensão, com pequenas quedas d'águas ao longo do seu curso, sendo a maior delas com cerca de $10 \mathrm{~m}$ de altura e desemboca diretamente no Rio Caí. O Ponto amostrado constitui-se em um ambiente de corredeira seguido por um poção. $\mathrm{O}$ trecho de corredeira apresenta pouca profundidade, alto grau de sombreamento devido à mata ciliar e leito constituído por rochas. O trecho de poção é menos sombreado, a correnteza é lenta, a profundidade média é de $1,2 \mathrm{~m}$, e o leito apresenta grande quantidade de matéria orgânica acumulada $\left(29^{\circ} 18^{\prime} 32^{\prime \prime} \mathrm{S}\right.$ e $50^{\circ} 45^{\prime} 41$ ' W).

- Açude 1 (ponto P6) - Foi construído em 1968, possui 4 ha de área e profundidade média estimada de 1,3 m. A água provém do arroio Tiririca e de nascentes próximas à área industrial do município de Canela, à áreas com aglomerações residenciais, 


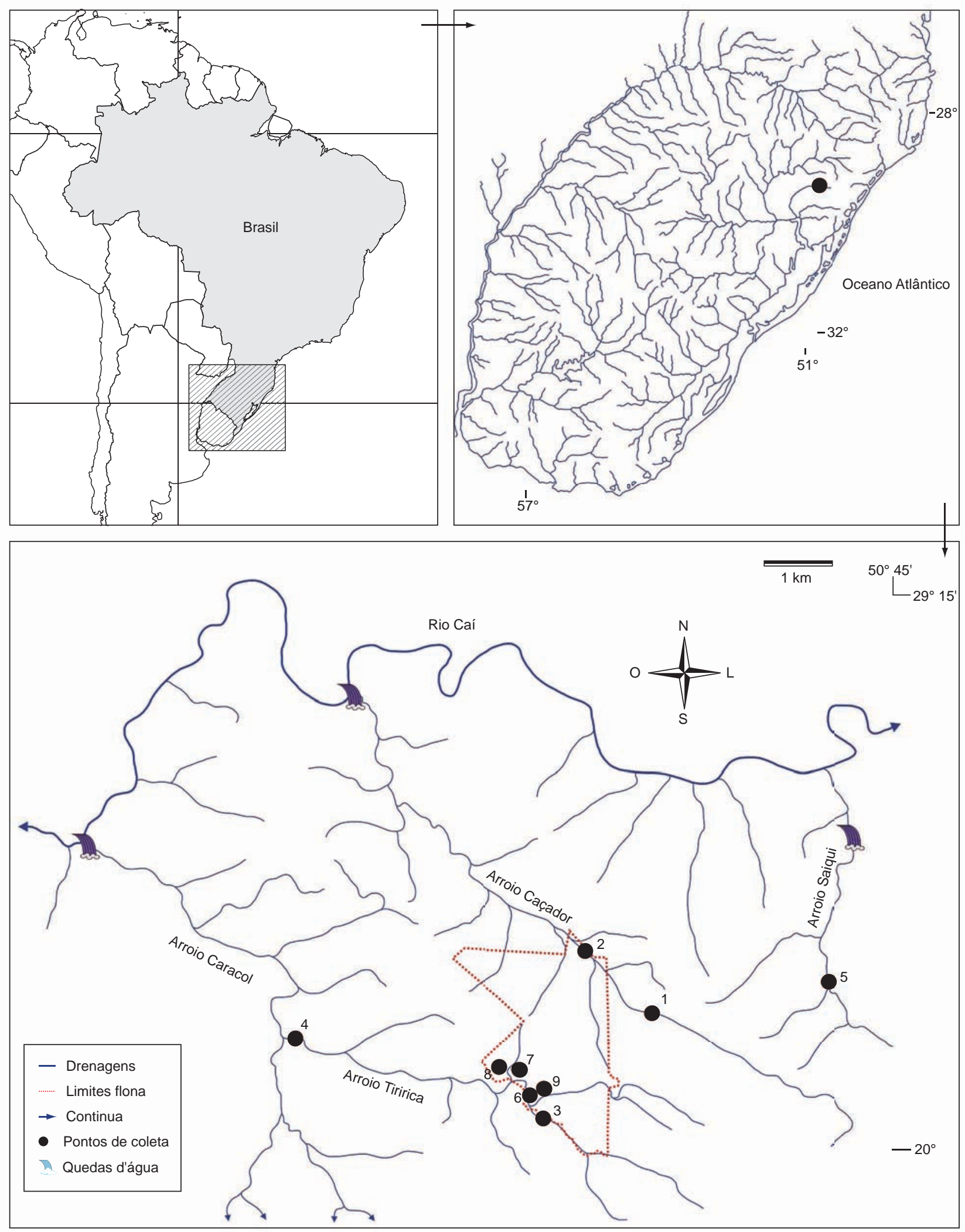

Figura 1. Localização dos pontos de coleta (P1 a P9) na Floresta Nacional de Canela e entorno, bacia do Rio Caí, RS. A linha vermelha tracejada indica a delimitação da área da unidade de conservação.

Figure 1. Location of the collection sites (P1 to P9) at Floresta Nacional de Canela and in the nearby area, Caí river basin, RS. The red traced line indicates the area of the conservation unity. 
à rodovia RS-235 e à localidade Saiqui, além de pequenos cursos d'água do interior da unidade. Ocorrem macrófitas aquáticas (Nymphoides indica, Nymphaea sp. e Myriophyllum sp.) cobrindo quase que totalmente a superfície da água (29 19' 27' S e 50॰48' 50” W).

- Açude 2 (ponto P7) - Sua construção foi concluída no ano de 2000, possui cerca de 3 ha de área e profundidade média estimada de $1,8 \mathrm{~m}$. A água provém de nascentes situadas no interior da unidade. Não apresenta macrófitas aquáticas cobrindo a superfície da água (29 19' 16" S e 50 48' 54” W).

- Açude 3 (ponto P8) - Foi construído em 1962, possui cerca de 1,5 ha de área e profundidade média estimada de 1,7 m. Sua água é oriunda de pequenos cursos d'água próximos a unidade. Ocorrem macrófitas aquáticas apenas nas margens do açude (29॰19’ 20”' S e 50॰ 49' 05” W).

- Banhado (ponto P9) - Localiza-se na margem do Açude 1, possui uma área aproximada de 2 ha e vegetação emergente constituída predominantemente por gravatás (Eryngium sp.) $\left(29^{\circ} 19^{\prime} 32^{\prime}\right.$ 'S e $50^{\circ} 48^{\prime} 37^{\prime}$ 'W).

\section{Métodos de amostragem}

Para a realização das coletas foram efetuadas 26 expedições a campo (com duração de um a 4 dias cada), no período de maio de 2004 a julho de 2007, contemplando todas as estações de cada ano. Os métodos aplicados variaram de acordo com o ambiente amostrado. Para a captura dos exemplares, nos arroios, foram empregados puçá (malha $2 \mathrm{~mm}$ entre nós opostos), aplicado tanto nas margens quanto junto ao leito, armadilhas do tipo covo (confeccionadas de garrafas PET) e arrastos com rede do tipo picaré (malha de $5 \mathrm{~mm}$ entre nós opostos). Nos açudes, foram empregadas redes de emalhar (malhas de 1,5 a 4,5 cm entre nós opostos), linhas de espera, puçá (aplicado junto à vegetação marginal) e armadilhas do tipo covo. No banhado, utilizou-se apenas puçá para a captura dos exemplares.

Os espécimes coletados foram fixados em formol $10 \%$ e, posteriormente, transferidos para álcool 70\%. Exemplares testemunhos foram depositados na coleção ictiológica do Museu de Ciências Naturais da Fundação Zoobotânica do Rio Grande do Sul (MCN FZB/RS), lotes MCN 18650 a 18665 e 18670 a 18673.

\section{Resultados}

Ao total, incluindo todos os pontos amostrados, foram encontradas 20 espécies d e peixes pertencentes a 10 famílias e 6 ordens (Tabela 1). As ordens Characiformes (com 6 espécies), Perciformes (com cinco espécies) e Siluriformes (com 4 espécies) foram as mais representativas, perfazendo $75 \%$ das espécies amostradas. Já as ordens Cyprinodontiformes e Gymnotiformes foram as menos representativas, com apenas uma espécie (5\%) cada.

\section{Distribuição de espécies por ambiente}

Para os açudes, foi registrado um total de 12 espécies (pontos P6, P7 e P8), sendo as ordens Perciformes (com cinco espécies) e Cypriniformes (com 3 espécies) as mais representativas. Os açudes caracterizaram-se pela predominância de ciclídeos (Australoheros sp., Crenicichla lepidota, Geophagus brasiliensis e Gymnogeophagus rhabdotus) e de espécies exóticas introduzidas, com distribuição restrita a estes ambientes (Ctenopharingodon idella, Cyprinus carpio, Hypophthalmichthys molitrix e Micropterus salmoides).

Somando os registros que ocorreram nos arroios (P1, P2, P3, P4 e P5), foram encontradas nove espécies em cursos d'água. Os Characiformes foram os mais representativos, com cinco espécies, seguidos por Siluriformes, com duas e Cyprinodontiformes e Perciformes ambas com uma. A riqueza específica no arroio Tiririca foi igual a 3 e no arroio Caçador, assim como no arroio Saiqui, igual a 6.

As espécies comuns aos 3 cursos d'água inventariados (Caçador, Tiririca e Saiqui) foram Hyphessobrycon luetkenii, Phalloceros caudimaculatus e Trichomycterus sp. Como espécies de ocorrência restrita ao arroio Caçador, foram registradas Astyanax aff. scabripinnis, Heptapterus mustelinus e Hyphessobrycon sp.. Por outro lado, Astyanax cremnobates e Astyanax jacuhiensis tiveram ocorrência apenas para o arroio Saiqui. Não ocorreram espécies restritas ao arroio Tiririca.

Hyphessobrycon luetkenii teve a distribuição mais ampla, ocorrendo em quase todos os pontos amostrados, com exceção do açude 3 (P8) e do banhado (P9). Por outro lado, Callichthys callichthys foi registrado somente no banhado $(\mathrm{P} 9)$.

\section{Discussão}

A predominância de ciclídeos, encontrada nos açudes inventariados neste estudo, pode ser explicada por esses ambientes possuírem características de água parada. Segundo Kullander (2003), a maioria dos ciclídeos neotropicais tem preferência por ambientes lênticos, com exceção de poucos gêneros. No trecho superior da bacia do Rio Caí, existe uma grande quantidade de açudes e barragens utilizados para piscicultura. O represamento de arroios e rios para construção de açudes ou barragens transforma um trecho de água corrente natural em um ambiente de água parada, podendo favorecer a proliferação de determinados organismos, como muitos ciclídeos neotropicais. Ao mesmo tempo, não se pode descartar a possibilidade de introdução intencional (peixamento) de algumas destas espécies de ciclídeos, visando o incremento da pesca na região.

Para os arroios, a maior representatividade das ordens Characiformes e Siluriformes está de acordo com o padrão encontrado na maioria dos rios e riachos neotropicais (Lowe-McConnell 1999). Mais de $80 \%$ das espécies de peixes continentais da América do Sul pertence às ordens Characiformes e Siluriformes (Castro 1999), as quais são representadas, principalmente, pelas famílias Characidae e Loricariidae, respectivamente (Reis et al. 2003a). Como esperado, nos arroios estudados predominaram espécies de caracídeos, entre os Characiformes. Por outro lado, nenhuma espécie da família Loricariidae foi encontrada, entre os Siluriformes. Nos arroios, esta ordem foi representada exclusivamente por Heptapterus mustelinus (Heptapteridae) e por Trichomycterus sp. (Trichomycteridae). Tais espécies apresentam o corpo cilíndrico e alongado, sugerindo uma adaptação à ocupação de pequenas fendas, como entre as pedras do leito dos ambientes de correnteza amostrados neste trabalho.

A baixa riqueza específica dos riachos em questão pode estar relacionada ao fato destes ambientes estarem localizados em uma região de cabeceira (a cerca de $800 \mathrm{~m}$ de altitude), limitados por quedas d'água. No estudo de Vieira et al. (2005), em riachos de cabeceira nas bacias do Rio Doce e São Francisco (MG), foram encontradas 43 espécies de peixes para os trechos abaixo de $750 \mathrm{~m}$ de altitude, 14 para os trechos de 750 a $850 \mathrm{~m}$ e apenas duas para os trechos acima de $850 \mathrm{~m}$ de altitude, isolados por quedas d'água.

No Rio Grande do Sul, Vilella et al. (2004), estudando a fauna aquática de um riacho costeiro da bacia hidrográfica do Rio Maquiné, encontraram zero, uma e 14 espécies de peixes em 3 pontos localizados a 730, 309 e $140 \mathrm{~m}$ de altitude, respectivamente, estando os 2 primeiros pontos isolados por cachoeiras. A existência de quedas d'água nos trechos superiores das drenagens é um dos principais fatores que dificultam a troca de fauna com os trechos inferiores, contribuindo, assim, para o isolamento das populações (Vilella et al. 2004, Vieira et al. 2005). De acordo com Peres-Neto et al. (1995), esse reduzido número de espécies em regiões de cabeceira pode, também, 
Tabela 1. Relação das espécies de peixes registradas (assinaladas por um X) em cada um dos pontos amostrados (P) na Floresta Nacional de Canela e região de entorno, RS, entre 2004 e 2007.

Table 1. List of the fish species registered (marked by an X) in each sampled site (P) at Floresta Nacional de Canela and in the nearby region, RS, from 2004 to 2007.

\begin{tabular}{|c|c|c|c|c|c|c|c|c|c|}
\hline Táxon / Ponto & P1 & $\mathbf{P 2}$ & P3 & $\mathbf{P 4}$ & P5 & P6 & P7 & P8 & P9 \\
\hline \multicolumn{10}{|l|}{ CHARACIFORMES } \\
\hline \multicolumn{10}{|l|}{ CHARACIDAE } \\
\hline Astyanax aff. scabripinnis (Jenyns, 1842) & $\mathrm{X}$ & $\mathrm{X}$ & & & & & & & \\
\hline Astyanax cremnobates Bertaco \& Malabarba, 2001 & & & & & $\mathrm{X}$ & & & & \\
\hline Astyanax jacuhiensis (Cope, 1894) & & & & & $\mathrm{X}$ & & & & \\
\hline Hyphessobrycon luetkenii (Boulenger, 1887) & $\mathrm{X}$ & $\mathrm{X}$ & $\mathrm{X}$ & $\mathrm{X}$ & $\mathrm{X}$ & $\mathrm{X}$ & $\mathrm{X}$ & & \\
\hline Hyphessobrycon sp. & $\mathrm{X}$ & & & & & & & & \\
\hline \multicolumn{10}{|l|}{ ERYTHRINIDAE } \\
\hline Hoplias malabaricus (Bloch, 1794) & & & & & & $\mathrm{X}$ & & & \\
\hline \multicolumn{10}{|l|}{ SILURIFORMES } \\
\hline \multicolumn{10}{|l|}{ HEPTAPTERIDAE } \\
\hline Heptapterus mustelinus (Valenciennes, 1835) & $\mathrm{X}$ & & & & & & & & \\
\hline Rhamdia quelen (Quoy \& Gaimard, 1824) & & & & & & $\mathrm{X}$ & $\mathrm{X}$ & $\mathrm{X}$ & \\
\hline \multicolumn{10}{|l|}{ CALLICHTHYIDAE } \\
\hline Callichthys callichthys (Linnaeus, 1758) & & & & & & & & & $\mathrm{X}$ \\
\hline \multicolumn{10}{|l|}{ TRICHOMYCTERIDAE } \\
\hline Trichomycterus sp. & $\mathrm{X}$ & $\mathrm{X}$ & & $\mathrm{X}$ & $\mathrm{X}$ & & & & \\
\hline \multicolumn{10}{|l|}{ PERCIFORMES } \\
\hline \multicolumn{10}{|l|}{ CICHLIDAE } \\
\hline Australoheros sp. & & & & & & $\mathrm{X}$ & $\mathrm{X}$ & $\mathrm{X}$ & \\
\hline Crenicichla lepidota Heckel, 1840 & & & & & & $\mathrm{X}$ & & & \\
\hline Geophagus brasiliensis (Quoy \& Gaimard, 1824) & & & & & $\mathrm{X}$ & $\mathrm{X}$ & $\mathrm{X}$ & $\mathrm{X}$ & \\
\hline Gymnogeophagus rhabdotus (Hensel, 1870) & & & & & & $\mathrm{X}$ & $\mathrm{X}$ & & \\
\hline \multicolumn{10}{|l|}{ CENTRARCHIDAE } \\
\hline *Micropterus salmoides (Lacepède, 1802) & & & & & & & $\mathrm{X}$ & & \\
\hline \multicolumn{10}{|l|}{ CYPRINIFORMES } \\
\hline \multicolumn{10}{|l|}{ CYPRINIDAE } \\
\hline *Ctenopharyngodon idella (Valenciennes, 1844) & & & & & & & $\mathrm{X}$ & & \\
\hline *Cyprinus carpio Linnaeus, 1758 & & & & & & & & $\mathrm{X}$ & \\
\hline *Hypophthalmichthys molitrix (Valenciennes, 1844) & & & & & & & & $\mathrm{X}$ & \\
\hline \multicolumn{10}{|l|}{ CYPRINODONTIFORMES } \\
\hline \multicolumn{10}{|l|}{ POECILIIDAE } \\
\hline Phalloceros caudimaculatus (Hensel, 1868) & $\mathrm{X}$ & $\mathrm{X}$ & & $\mathrm{X}$ & $\mathrm{X}$ & & & & \\
\hline \multicolumn{10}{|l|}{ GYMNOTIFORMES } \\
\hline \multicolumn{10}{|l|}{ GYMNOTIDAE } \\
\hline Gymnotus aff. carapo Linnaeus, 1758 & & & & & & & $\mathrm{X}$ & & \\
\hline
\end{tabular}

\footnotetext{
*Espécie exótica introduzida.
}

estar relacionado às altas flutuações ambientais a qual esses ambientes são submetidos e a baixa diversidade de hábitats disponíveis.

Segundo a análise de Castro (1999), o predomínio de espécies de peixes de pequeno porte (de 80 a $100 \%$ ), é um padrão provável para riachos sul-americanos. Os resultados obtidos para os arroios Caçador, Saiqui e Tiririca são similares a este padrão esperado, uma vez que $78 \%$ das espécies capturadas possuem pequeno porte (menor que $15 \mathrm{~cm}$ ). Conforme ainda Castro (1999), os ambientes de riachos parecem ter exercido, ao longo da evolução, uma série de pressões seletivas que favoreceram a redução no tamanho corporal das espécies de peixes que utilizam estes ambientes.

Em relação aos resultados obtidos dos riachos inventariados, destaca-se a ocorrência de espécies com "status" taxonômico inde- finido, tais como $A$. aff. scabripinnis, a qual pertence a um complexo de espécies com o corpo morfologicamente parecido (Bertaco \& Malabarba 2001, Bertaco \& Lucena 2006), de Trichomycterus sp., uma espécie ainda não descrita, e de Australoheros sp., espécie provavelmente não descrita, cuja identificação taxonômica carece de uma revisão do gênero para o sistema da laguna do Patos. Hyphessobrycon sp. também parece ser uma espécie diferente das já descritas para o sistema da laguna dos Patos, porém cabe ressaltar que apenas um exemplar foi coletado, mesmo após um aumento no esforço de captura. Ainda é importante destacar o registro de $A$. cremnobates, espécie com distribuição conhecida apenas para regiões de elevada altitude da Serra Geral do RS (de 800 à $1000 \mathrm{~m}$ ), nas cabeceiras de tributários dos rios Jacuí e Maquiné (Bertaco \& Malabarba 2001). 
Embora neste levantamento as espécies exóticas tenham sido documentadas apenas em açudes, já existem publicações demonstrando a ocorrência de peixes não nativos em ambientes naturais do sistema da laguna dos Patos (e.g. Braun et al. 2003, Garcia et al. 2004, SaccolPereira et al. 2006). A introdução de espécies é considerada uma das maiores causas para perda de biodiversidade na atualidade (Fontana et al. 2003, Ziller \& Zalba 2007). Peixes exóticos em ambientes naturais podem agir como predadores, competidores ou ainda disseminar organismos patogênicos (Reis et al. 2003b). Desse modo, a erradicação das espécies exóticas da unidade de conservação é altamente recomendável e de fundamental importância para a preservação dos peixes nativos e dos ecossistemas aquáticos da região.

Os impactos ambientais já exercidos sobre a área inventariada podem ter ocasionado uma diminuição da biodiversidade de peixes. No entanto, a falta de estudos prévios inviabiliza que sejam feitas maiores considerações em relação às possíveis modificações ocorridas na ictiofauna do local.

\section{Agradecimentos}

Aos colegas do Centro Universitário Feevale que participaram da realização do projeto que resultou neste trabalho, ao José Pezzi da Silva (PUCRS) e Paulo César Milani (PUCRS) pelo auxílio nas identificações dos peixes, ao Marco A. Azevedo pelas sugestões do texto e ao Daniel Bühler e Tassiana G. de Paula pela ajuda nas coletas. Um agradecimento especial ao Ewerton Ferraz, Raul Paixão Coelho e Paulo Rossi (ICMBio/Canela), por todo apoio logístico e cordialidade oferecidos durante as expedições a Floresta Nacional de Canela.

\section{Referências Bibliográficas}

AGOSTINHO, A.A., THOMAZ, S.M. \& GOMES, L.C. 2005. Conservação da biodiversidade em águas continentais do Brasil. Megadiversidade, 1(1):70-78.

BECKER, F.G., GROSSER, K.M., MILANI, P.C.C. \& BRAUN, A.S. 2006. Peixes. In Regiões da Lagoa do Casamento e dos Butiazais de Tapes, Planície Costeira do Rio Grande do Sul (F.G. Becker, R.A. Ramos, L.A. Moura, orgs). MMA; SBF, Brasília, p. 262-275.

BERTACO, V.A. \& LUCENA, C.A.S. 2006. Two new species of Astyanax (Ostariophysi: Characiformes: Characidae) from eastern Brazil with a synopsis of the Astyanax scabripinnis species complex. Neotrop. Ichthyol. 4(1):53-60.

BERTACO, V.A. \& MALABARBA, L.R. 2001. Description of two new species of Astyanax (Teleostei: Characidae) from headwater streams of Southern Brazil, with comments on the "A. scabripinnis species complex". Ichthyol. Explor. Fres. 12(3):221-234.

BÖHLKE, J.E., WEITZMAN, S.H. \& MENEZES, N.A. 1978. Estado atual da sistemática de peixes de água doce da América do Sul. Acta Amazon. 8(4):657-677

BRAUN, A.S., MILANI, P.C.C. \& FONTOURA, N.F. 2003. Registro da introdução de Clarias gariepinus (Siluriformes, Clariidae) na laguna dos Patos. Biociências, 11(1):101-102.

CASTRO, R.M.C. 1999. Evolução da ictiofauna de riachos sul-americanos: padrões gerais e possíveis processos causais. In Ecologia de Peixes de Riachos (E.P. Caramaschi, R. Mazzoni \& P.R. Peres-Neto, eds). PPGEUFRJ, Rio de Janeiro, p. 139-155. Série Oecologia Brasiliensis, v. 6.

FERRAZ, E.A.R. 2003. Meio Ambiente em Canela: Floresta Nacional IBAMA. In Raízes de Canela (P. Oliveira \& V.L.M. Barroso, orgs). Editora EST, Porto Alegre, p. 423-432.

FONTANA, C.S., BENCKE, G.A. \& REIS, R.E. 2003. Livro vermelho da fauna ameaçada de extinção no Rio Grande do Sul. Edipucrs, Porto Alegre.

FORTES, A.B. 1959. Geografia física do Rio Grande do Sul. Editora Globo, Porto Alegre.

GARCIA, A.M., LOEBMANN, D., VIEIRA, J.P. \& BEMVENUTI, M.A. 2004. First records of introduced carps (Teleostei, Cyprinidae) in the natural habitats of Mirim and Patos Lagoon estuary, Rio Grande do Sul, Brazil. Rev. Bras. Zool. 21(1):157-159.

HENSEL, R.F. 1868. Beiträge zur kenntniss der Wirbeltiere Süd-Brasiliens. Arch. Naturgesch. 34:356-375.
HENSEL, R.F. 1870. Beiträge zur kenntniss der Wirbeltiere Süd-Brasiliens. Arch. Naturgesch. 36:50-91.

KULLANDER, S.O. 2003. Family Cichlidae. In Check list of the freshwater fishes of South and Central America (R.E. Reis, S.O. Kullander \& C.J. Ferraris Jr., eds). Edipucrs, Porto Alegre, p. 605-654.

LOWE-McCONNELL, R.H. 1999. Estudos ecológicos de comunidades de peixes tropicais. Editora da Universidade de São Paulo, São Paulo.

MORENO, J.A. 1961. Clima do Rio Grande do Sul. Secretaria da Agricultura do Rio Grande do Sul, Porto Alegre.

PERES-NETO, P.R., BIZERRIL, C.R.F. \& IGLESIAS, R. 1995. An overview of some aspects of river ecology: a case study on fish assemblages distribution in an eastern Brazilian coastal river. Oecol. Bras. 1:317-334.

REIS, R.E., KULLANDER, S.O. \& FERRARIS Jr, C.J. 2003a. Check list of the freshwater fishes of South and Central America. Edipucrs, Porto Alegre.

REIS, R.E., LUCENA, Z.M.S., LUCENA, C.A.S. \& MALABARBA, L.R. 2003b. Peixes. In Livro vermelho da fauna ameaçada de extinção no Rio Grande do Sul (C.S. Fontana, G.A. Bencke, R.E. Reis, eds). Edipucrs, Porto Alegre, p. 117-145.

SACCOL-PEREIRA, A., MILANI, P.C.C. \& FIALHO, C.B. 2006. Primeiro registro de Acestrorhynchus pantaneiro Menezes, 1992 (Characiformes, Acestrorhynchidae) no sistema da laguna dos Patos, Rio Grande do Sul, Brasil. Biota Neotrop. 6(3): http://www.biotaneotropica.org.br/v6n3/ pt/abstract?short-communication+bn00706032006 (último acesso em 18/08/2008).

SCHAEFER, S.A. 1998. Conflict and resolution: impact of new taxa on Phylogenetic studies of the Neotropical cascudinhos (Siluroidei: Loricariidae). In Phylogeny and classification of Neotropical fishes (L.R. Malabarba, R.E. Reis, R.P. Vari, Z.M.S. Lucena \& C.A.S. Lucena, eds). Edipucrs, Porto Alegre, p. 375-400.

SCHNEIDER, P.R., BRENA, D., FINGER, C., LONGHI, S., HOPPE, J., VINADÉ, L., NASCIMENTO, R., AZAMBUJA, P. \& MENEZES, L. 1989. Plano de manejo para Floresta Nacional de Canela. FATEC, Santa Maria.

Secretaria do Meio Ambiente do Estado do Rio Grande do Sul - SEMA/RS. Sistema Estadual de recursos hídricos. Disponível em: http://www.sema. rs.gov.br/sema/jsp/rechidro.jsp (último acesso em 20/07/2008).

Sistema Nacional de Unidades de Conservação - SNUC. 2002. Lei no. 9.985 , de 18 de julho de 2000; decreto no. 4.430, de 22 de agosto de 2002. Sistema Nacional de Unidades de Conservação da Natureza: 2 ed. MMA/SBF, Brasília.

TEIXEIRA, M.B., COURA-NETO, A.B., PASTORE, U. \& RANGEL-FILHO, A.L.R. 1986. Vegetação: as regiões fitoecológicas, suas naturezas e seus recursos econômicos e estudo fitogeográfico. In Levantamento de recursos naturais, Folha SH-22 Porto Alegre e parte das folhas SH-21 Uruguaiana e SI-22 Lagoa Mirim. IBGE; Fundação Instituto de Geografia e Estatística, Rio de Janeiro. Projeto RADAM Brasil, v. 33, 79 p.

TEIXEIRA, R.L. 1989. Aspectos da ecologia de alguns peixes do arroio Bom Jardim, Triunfo-RS. Rev. Bras. Biol. 49(1):183-192.

VARI, R.P. \& MALABARBA, L.R. 1998. Neotropical ichthyology: an overview. In Phylogeny and classification of Neotropical fishes (L.R. Malabarba, R.E. Reis, R.P. Vari, Z.M.S. Lucena \& C. A. S. Lucena, eds). Edipucrs, Porto Alegre, p. 1-12.

VIEIRA, F., SANTOS, G.B. \& ALVES, C.B. 2005. A ictiofauna do Parque Nacional da Serra do Cipó (Minas Gerais, Brasil) e áreas adjacentes. Lundiana, 6(suppl.):77-87.

VILELLA, F.S., BECKER, F.G., HARTZ, S.M. \& BARBIERI, G. 2004. Relation between environmental variables and aquatic megafauna in a first order stream of the Atlantic Forest, southern Brazil. Hydrobiologia, 528:17-30.

VOLKMER-RIBEIRO, C., GUADAGNIN, D.L., ROSA-BARBOSA, R., SILVA, M.M., DRÜGG-HAHN, S., LOPES-PITONI, V.L., GASTAL, H.A.O., BARROS, M.P. \& DEMAMAN, L.V. 2004. A polyethylenetherephthalate (pet) device for sampling freshwater benthic macroinvertebrates. Braz. J. Biol. 64(3A):531-541.

ZILLER, S.R. \& ZALBA, S. 2007. Propostas de ação para prevenção e controle de espécies exóticas invasoras. Natur. Conserv. 5(2):8-15. 